\title{
How to protect factory workers from COVID-19?
}

Dina M. Elshenawi * and Yuefen Wang *

Coronavirus has become a very serious global health problem. Since December 2019, a number of new cases of patients with pneumonia caused by the Coronavirus disease have been found in Wuhan City, Hubei Province, China. With the global spread of the epidemic, other types of cases have been found in other regions of China and abroad. Factory workers play an important and essential role in the production of goods needed by society. Protecting them is essential to maintain the stability of factory productivity. This editorial provides guidance in how to protect factory workers from COVID-19 infection.

Keywords: COVID-19; Factory workers; Mask; Productivity; Wood; Wuhan pneumonia

Contact information: School of Marxism, Northeast Forestry University, Harbin, P.R. China 150040;

*Corresponding authors:dr_dina@nefu.edu.cn; mlbwyf@126.com,wangyuefen@yahoo.com

\section{Geographical Distribution of COVID-19}

Coronavirus has become a global epidemic, i.e. a pandemic. By the end of December 2019, a number of patients with pneumonia caused by unknown etiology had been reported in Wuhan City, Hubei Province, China (Cui et al 2019; Hui et al 2020; Nishiura et al. 2020; Liu et al. 2020). According to Xu Jianguo, a Chinese expert, results carried out from their lab showed that a new-type of Coronavirus (2019-nCov) had caused the viral pneumonia in Wuhan (Xinhua 2020). The 2019-nCoV exhibits marked differences in genome sequence from the SARS Coronavirus (SARS-CoV) and MERS-CoV (Xinhua 2020; Huaxia 2020; Liu et al. 2020). On 11 February 2020, the World Health Organization (WHO) gave 2019-nCoV a new official name as Coronavirus Disease 2019 (COVID-19) (Chen 2020).

Globally, as of 10:00 CET 1 May 2020, WHO has reported a total of 3,175,207 confirmed cases and 224,172 deaths (WHO). There are 1,291,917 confirmed cases and 69,087 deaths in the region of the Americas. There are 1,461,404 confirmed cases and 138,200 deaths in the European region. There are 148,838 confirmed cases and 6,127 deaths in the Western Pacific region.

A recent editorial by Liu et al (2020) stated that "there are negative effects of the COVID-19 on people's daily lives and factory production." On the other hand, there are some factories that are fulfilling important needs such as wood products and medical supplies, and they have been kept open. As we know, wood lumber could be used for building beds for hospitals to receive an overflow of patients, because of the increase in the number of patients during the spread of COVID-19.

As we know, factory workers play an important and essential role in the production processes. Protecting them is essential to maintaining the stability and productivity of any factory. This editorial considers how to protect factory workers from COVID-19. 


\section{How to protect factory workers from COVID-19}

Does wearing a mask mean factory workers have the COVID-19? No, it's just an efficient way to protect themselves if they are wearing it correctly! At the time of writing this paper, COVID-19 has already infected more than 3,000,000 people across the world. Reports suggest a correlation between widespread use of facial masks in a country and lower rates of the spread of COVID-19. For instance, although Japan has a larger population than Italy (Worldometers), Italy has more people that are infected with COVID19 than Japan. Japanese people began to wear masks as soon as COVID-19 had been reported in Japan. On the other hand, unfortunately, Italians were late to wear masks when they went outside. As of 10:00 CET 1 May 2020, there are 205,463 confirmed and 27,967 deaths in Italy (WHO). On the other hand, there are 14,281 confirmed and 432 deaths in Japan (WHO). Information of this type provides strong evidence that wearing a mask is very important in saving lives during this critical time.

So how can factory workers protect themselves from this deadly virus? They have to wash their hands frequently for sure, and they do need to wear a mask when they go outside, especially in crowded areas and factories.

When a factory worker needs to wear a mask, he or she should follow the steps below, as also shown in Fig. 1:

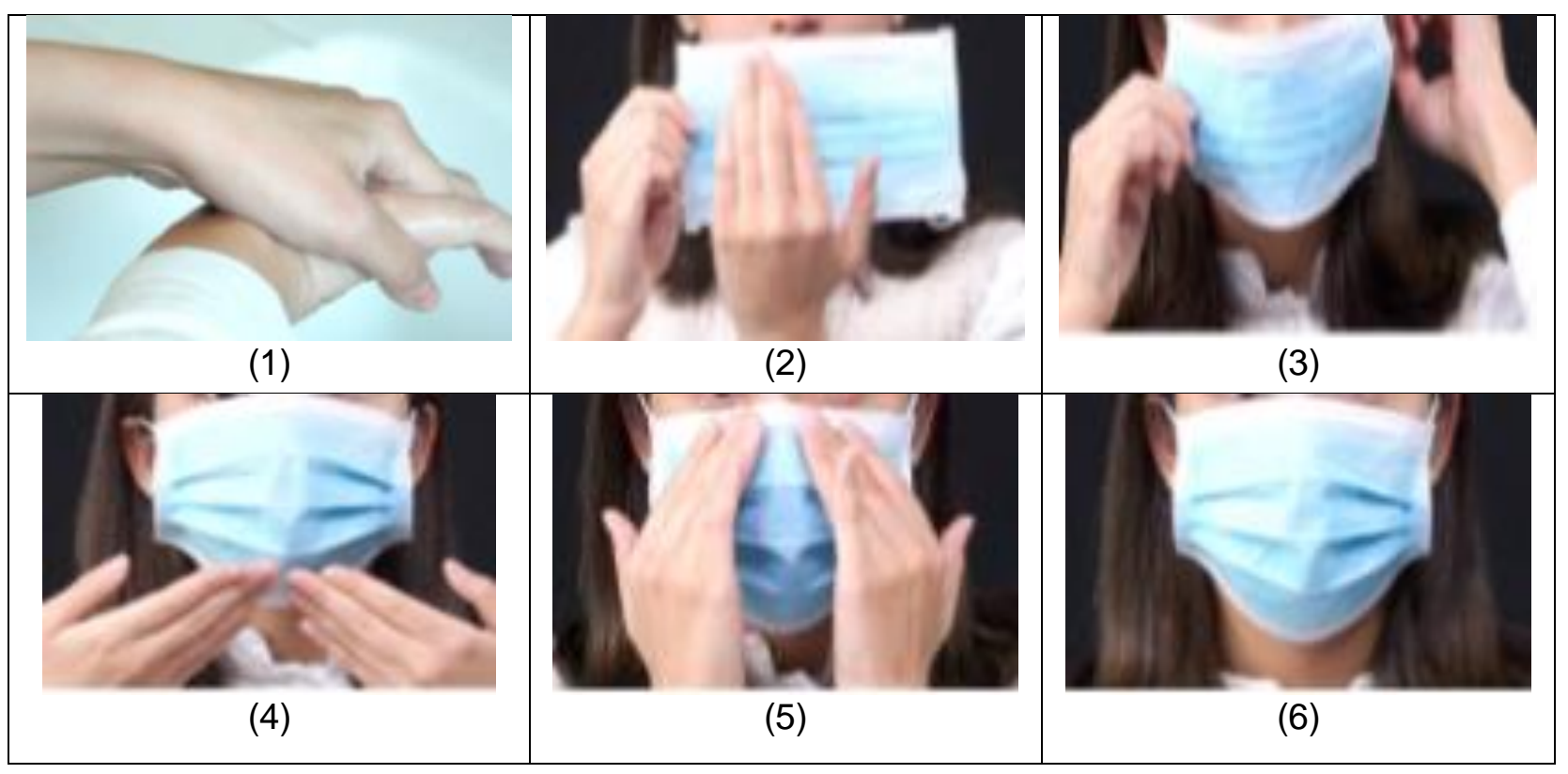

Fig. 1. Mask prevention: The right way to wear a mask

1. He or she should wash hands before wearing surgical masks. To achieve the best bactericidal effect, he or she should squeeze enough rubbing alcohol hand sanitizer evenly on: palm, back of hand, finger gap, back of finger, thumb, fingertip, and wrist; 2. He or she should distinguish the front and back of surgical masks. Generally, the outside with color or folds should be facing downwards and the side with metal strips should be facing upwards;

3. If he or she chooses a hanging ear surgical mouth version, wrap the elastic band around the ears so that the mask is close to the face; 
4. He or she should open the surgical mask so that the mask completely covers the mouth, nose, and chin;

5. He or she should press the metal diaphragm of the surgical mask along both sides of the nose;

6. After wearing a mask, he or she should avoid touching the mask. If he or she must touch the mask, it is necessary to wash hands thoroughly before and after touching it.

After using a mask, the factory worker should follow the steps below (see Fig. 2):

1. After washing his hands, remove the elastic band of the mask from both ears.

2. Try to avoid touching the outer part of the mask, and fold the mask outward.

3. Put it in a compact bag and seal it.

4. Discard the removed mask in a covered trash can, and then clean hands immediately.

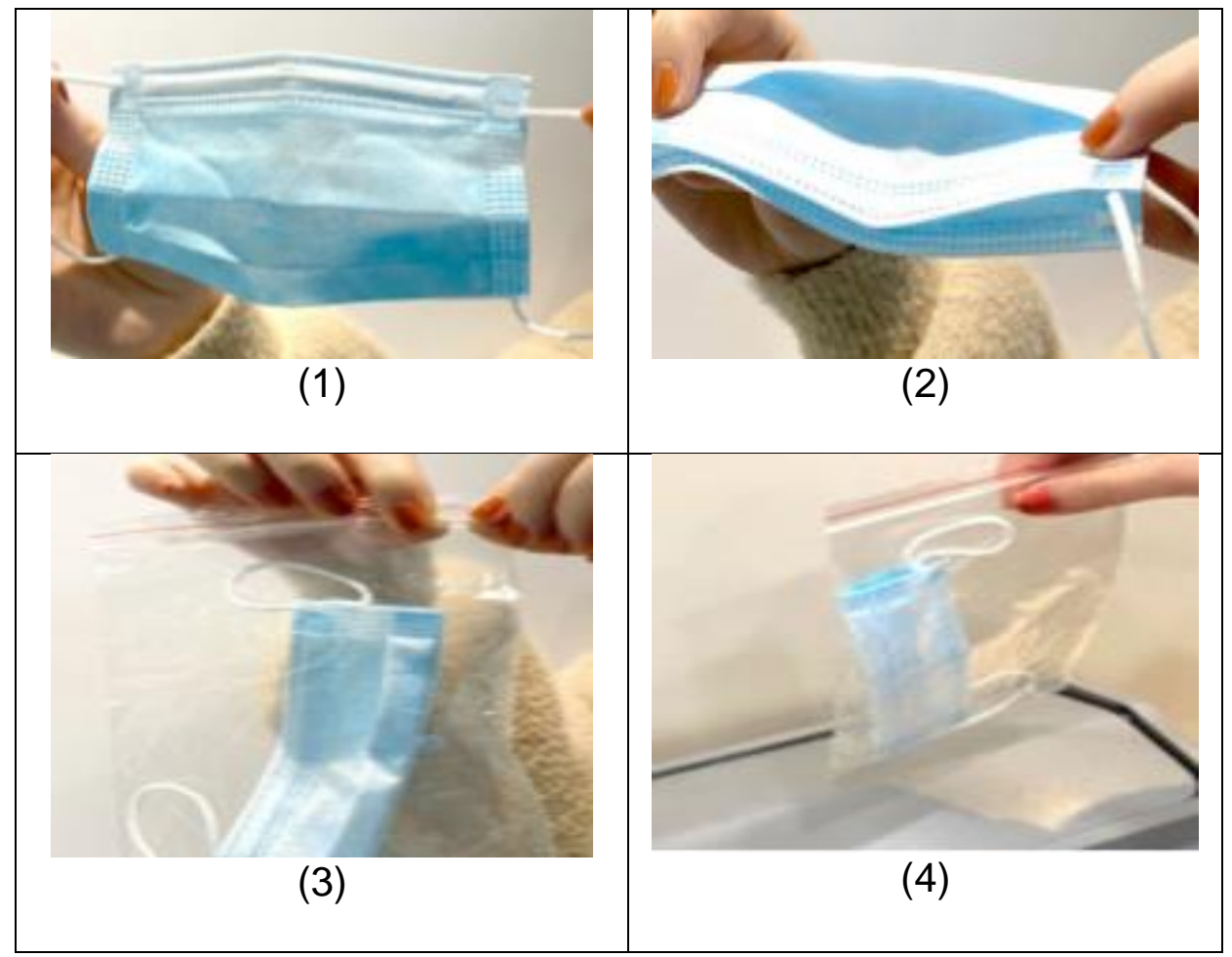

Fig. 2. Discard the Mask Properly

\section{Funding}

The authors are thankful for support from the Higher Education Reform Project of Heilongjiang Province: (Project number: SJGST2017001).

\section{References Cited}

Chen, W.-H., "Novel coronavirus named COVID-19 by WHO," Available online: http://global.chinadaily.com.cn/a/202002/11/WS5e42c999a310128217276c51.html 
Cui, J., Li, F., and Shi, Z. L. (2019). "Origin and evolution of pathogenic coronaviruses," Nature Reviews Microbiolog. 17, 181-192. DOI: 10.1038/s41579-018-0118-9

Huaxia, "New-type coronavirus causes pneumonia in Wuhan: Expert," Available online: http://www.xinhuanet.com/english/2020-01/09/c_138690570.htm (accessed on 21 January 2020)

Hui, D. S. I., Azhar, E., Madani, T. A., Ntoumi, F., Kock, R., Dar, O., Ippolito, G., McHugh, T. D., Memish, Z. A., Drosten, C., et al. (2020). "The continuing 2019nCoV epidemic threat of novel coronaviruses to global health-The latest 2019 novel coronavirus outbreak in Wuhan, China," International Journal of Infectious Diseases 91, 264-266. DOI: 10.1016/j.ijid.2020.01.009

Liu, K., Wang, H., Liu, H.-Y., Nie, S.-X., Du, H.-S., and Si, C.-L. (2020). "COVID-19: Challenges and perspectives for the pulp and paper industry worldwide," BioResources 15(3), 4638-4641.

Liu, S.-L., and Saif, L. (2020). "Emerging viruses without borders: The Wuhan Coronavirus," Viruses 12, 130. DOI: 10.3390/v12020130

Nishiura, H., Jung, S.-M., Linton, N. M., Kinoshita, R., Yang, Y., Hayashi, K., Kobayashi, T., Yuan, B., and Akhmetzhanov, A. R. (2020). "The extent of transmission of novel coronavirus in Wuhan, China," Journal of Clinical Medicine 9(2), 330. DOI: 10.3390/jcm9020330

Xinhua. (2020). "New-type coronavirus causes pneumonia in Wuhan: Expert," http://en.hubei.gov.cn/news/newslist/202001/t20200109_1906779.shtml

WHO (2020). https://www.who.int/docs/default-source/coronaviruse/situationreports/20200501-covid-19-sitrep.pdf?sfvrsn=742f4a18_2

Worldometers (2020) https://www.worldometers.info/world-population/ 